\title{
Methodology Report \\ Experimentally Approaching the ICU: Monitoring Outcome-Based Responses in the Two-Hit Mouse Model of Posttraumatic Sepsis
}

\author{
Susanne Drechsler, Katrin M. Weixelbaumer, Heinz Redl, Martijn van Griensven, \\ Soheyl Bahrami, and Marcin F. Osuchowski
} Ludwig Boltzmann Institute for Experimental and Clinical Traumatology in the Trauma Research Center of AUVA,
Donaueschingenstraße 13, 1200 Vienna, Austria

Correspondence should be addressed to Marcin F. Osuchowski, marcin.osuchowski@trauma.lbg.ac.at

Received 13 October 2010; Accepted 13 December 2010

Academic Editor: Monica Fedele

Copyright (C) 2011 Susanne Drechsler et al. This is an open access article distributed under the Creative Commons Attribution License, which permits unrestricted use, distribution, and reproduction in any medium, provided the original work is properly cited.

To simulate and monitor the evolution of posttraumatic sepsis in mice, we combined a two-hit model of trauma/hemorrhage (TH) followed by polymicrobial sepsis with repetitive blood sampling. Anesthetized mice underwent femur fracture/sublethal hemorrhage and cecal ligation and puncture (CLP) $48 \mathrm{~h}$ later. To monitor outcome-dependent changes in circulating cells/biomarkers, mice were sampled daily (facial vein) for 7 days and retrospectively divided into either dead (DIE) or surviving (SUR) by post-CLP day 7. Prior to CLP, AST was 3-fold higher in DIE, while all other post-TH changes were similar between groups. There was a significant post-CLP intergroup separation. In SUR, RBC and Hb were lower, platelets and neutrophils higher, and lymphocytes mixed compared to DIE. In DIE, all organ function markers except glucose (decrease) were few folds higher compared to SUR. In summary, the combination of daily monitoring with an adequate two-hit model simulates the ICU setting, allows insight into outcome-based responses, and can identify biomarkers indicative of death in the acute posttraumatic sepsis in mice.

\section{Introduction}

With more than 751.000 cases per annum in USA [1] and an incidence ranging from 0.4 to 1 case/1000 people in Europe [2], sepsis is the leading cause of death in noncoronary intensive care units [3]. Sepsis is routinely described as the body's systemic response to an invading pathogen such as bacteria and/or fungus [4]. The current consensus implies that early (acute) septic deaths have been associated with an excessive exacerbation of the proinflammatory immune response (termed systemic inflammatory response syndrome or SIRS), which is then followed by the compensatory anti-inflammatory response syndrome (CARS), although biomarkers typical to either of those syndromes may be released simultaneously $[5,6]$. Systemic spread of pathogens can originate from numerous organs/systems of the body-the lung representing the most frequent site of infection $(68 \%)$, followed by the abdomen (22\%), blood (20\%), and urinary tract (14\%) [7].

The pathophysiology of sepsis is further complicated by a variety of invading microorganisms, and that sepsis typically occurs as a secondary complication (second hit). Epidemiological data are inconsistent on the incidence of post-traumatic sepsis: existing studies demonstrated that post-injury sepsis/severe sepsis incidence ranges from $2 \%$ to over 30\% [8-10]. Furthermore, it has been corroborated that trauma patients with sepsis have significantly higher ICU admission rates and mortality than their non-septic counterparts, mostly due to the development of multiple organ dysfunction syndrome (MODS) [10,11].

Animal models are the launch platform to study the pathophysiology of sepsis and septic shock since they feature 


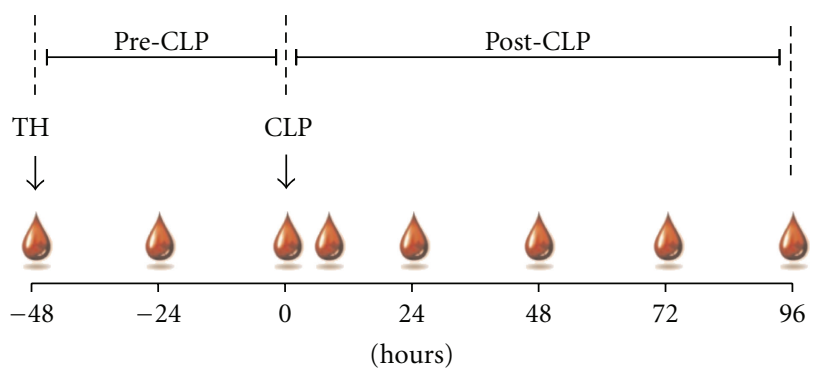

Blood sampling

FIgURE 1: Schematic of the two-hit model and repetitive blood sampling. 3-month-old female mice were subjected to trauma and hemorrhage (TH) followed by polymicrobial CLP-induced sepsis. Time span of model was divided into a pre-and post-CLP phase. Starting at TH, a $20 \mu \mathrm{L}$ blood sample was collected daily (until postTH day 7 post-CLP/day 5) from each animal, including one extra sample at $6 \mathrm{~h}$ after-CLP.

a maximally controlled milieu and optimal experimental reproducibility $[12,13]$. Over the past decades, the most widely used approach has typically employed a bolus administration of live/inactivated bacteria and their components such as lipopolysaccharide (LPS). While acute intravenous infusion of LPS/endotoxin is essentially inadequate for recapitulation of protracted septic events [14, 15], the cecal ligation and puncture (CLP) and colon ascendens stent peritonitis (CASP) models, although not ideal, appear to be the best existing alternative for emulating human polymicrobial sepsis to date $[16,17]$. Despite their wide use, all of the above-mentioned models mirror primary sepsis induced by a single challenge (one hit). To date, only a few animal models attempted to recapitulate another, more clinically relevant scenario of the two-hit challenge, in which sepsis develops shortly after a survivable traumatic event. An early twohit model consisting of an initial hemorrhage followed by a subsequent polymicrobial sepsis (CLP) in the mouse [18] was developed by Stephan and collaborators. This approach was further expanded by Van Griensven et al., who combined partially lethal hemorrhage and nonlethal trauma as the first hit and applied CLP as the subsequent hit [19]. A different two-hit approach, developed to investigate the evolution of nosocomial lung infections after abdominal sepsis in the mouse, features CLP (as the first hit) followed by a singlestrain bacterial pneumonia [20-22].

The multiorigin epidemiology of sepsis and dynamic alterations of its responses emphasize that successful treatment of sepsis should rely on highly individualized therapeutic interventions [23]. We have recently shown that dexamethasone treatment administered to homogenous subgroups of mice (stratified based on predicted outcome) markedly improved the survival of those mice with severe sepsis but conferred no benefit on the moderately sick cohort [24]. Such a targeted approach requires preclinical modeling that sufficiently reproduces both multistep pathophysiology of a given septic complication and the standard ICU monitoring protocol. Therefore, we combined the modified two-hit sepsis model (unilateral femur fracture/sublethal hemorrhage followed by CLP) with our recently validated daily blood-based monitoring method. Next, we tested this design by surveying the evolution of exemplary immunological, organ function, and metabolic alterations prior to and after intraabdominal sepsis. Specifically, differences between dying and surviving animals during both hit phases were investigated.

\section{Materials and Methods}

2.1. Animals. 3-month-old, female CD-1 mice (total $n=$ 120) with an average weight of $30 \mathrm{~g}$ were used for all experiments. Mice were purchased from Harlan Laboratories (Udine, Italy) and were kept in groups of five per cage on a $12 \mathrm{~h}$ light-dark cycle. Temperature was maintained between $22-24^{\circ} \mathrm{C}$, and standard rodent diet and water were provided ad libitum to all mice throughout the experiments. All animal procedures were approved by the local legislative committee and conducted according to National Institute of Health guidelines.

2.2. Two-Hit Model. We modified the original two-hit trauma/hemorrhage protocol developed by van Griensven et al. [19]. In brief, the first hit consisted of a noncomminuted, unilateral, midshaft femur fracture followed by a hemorrhage via retro-orbital puncture (Figure 1). Femur fracture was produced by custom-designed blunt pliers and confirmed by X-ray. For hemorrhage, total blood volume (TBV) was calculated as $6 \%$ of total body weight and both $50 \%$ and $40 \%$ of TBV were tested to achieve survival of at least $95 \%$ at the end $(0 \mathrm{~h})$ of the posttrauma/hemorrhage (TH) phase. After- $\mathrm{TH}$, mice were resuscitated with $0.9 \%$ sodium chloride subcutaneously, $(\mathrm{NaCl})$ with four times the volume of shed blood: $1 \mathrm{~mL}$ containing analgesia $(0.05 \mathrm{mg} / \mathrm{kg}$ buprenorphine, Temgesic) was administered immediately after hemorrhage (aimed to reproduce the phase of restricted resuscitation), while the remaining volume was given $1 \mathrm{~h}$ later (aimed to reproduce the phase of unrestricted resuscitation).

To induce the second hit, mice were subjected to CLP $48 \mathrm{~h}$ after-TH (Figure 1). We followed the original CLP protocol by Wichterman et al. [25] with a number of modifications specified elsewhere $[26,27]$. In order to receive an approximate mortality of $50 \%$ at post-CLP day 5 (postTH day 7), different grade of CLP severity was induced by increasing needle sizes (i.e., 20, 19, 18, or 17 gauge (G)). Starting with $\mathrm{TH}$, mice were resuscitated twice daily (excluding the immediate posthemorrhage resuscitation) with $1 \mathrm{ml}$ of $0.9 \% \mathrm{NaCl}$ including analgesia $(0.05 \mathrm{mg} / \mathrm{kg}$ buprenorphine, Temgesic). From $2 \mathrm{~h}$ after-CLP onward, every mouse was resuscitated twice daily for 5 days with $1 \mathrm{~mL}$ Ringer solution containing analgesia (as above) and widerange antibiotic $(25 \mathrm{mg} / \mathrm{kg}$ imipenem, Zienam) to emulate the "Surviving Sepsis Campaign Guidelines for Management of Severe Sepsis and Septic Shock" [28]. For all surgical procedures, mice were anesthetized with isoflurane (Forane), and survival was monitored for seven days after $\mathrm{TH}$. 


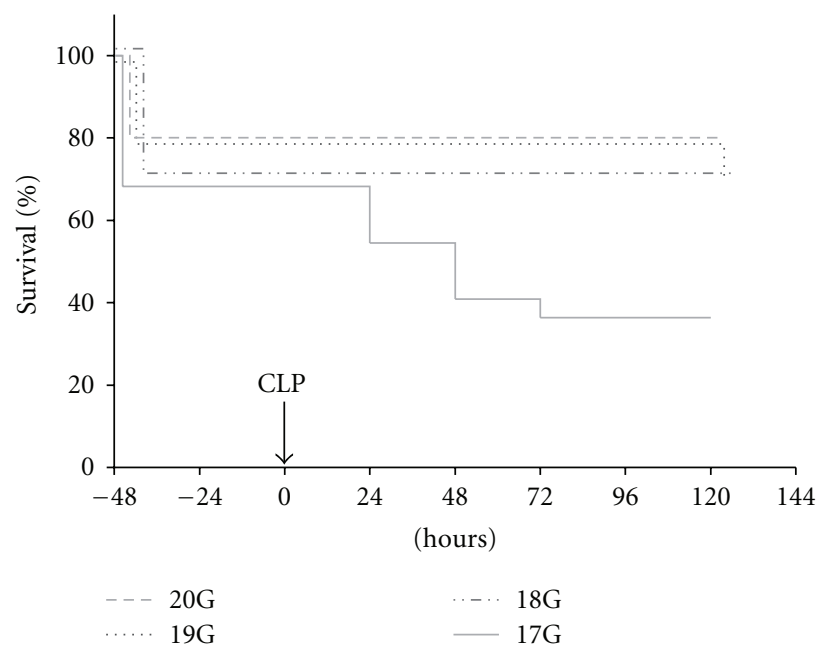

(a)

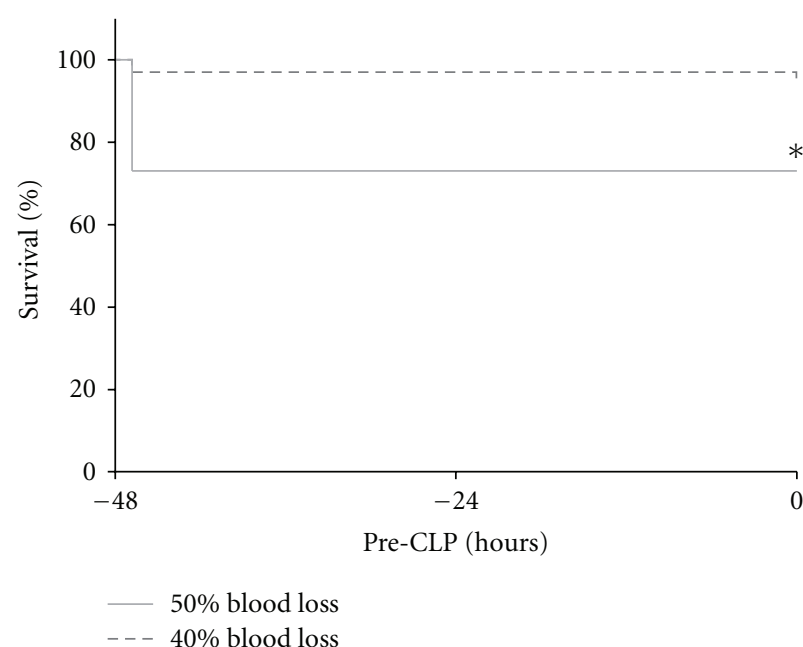

(b)

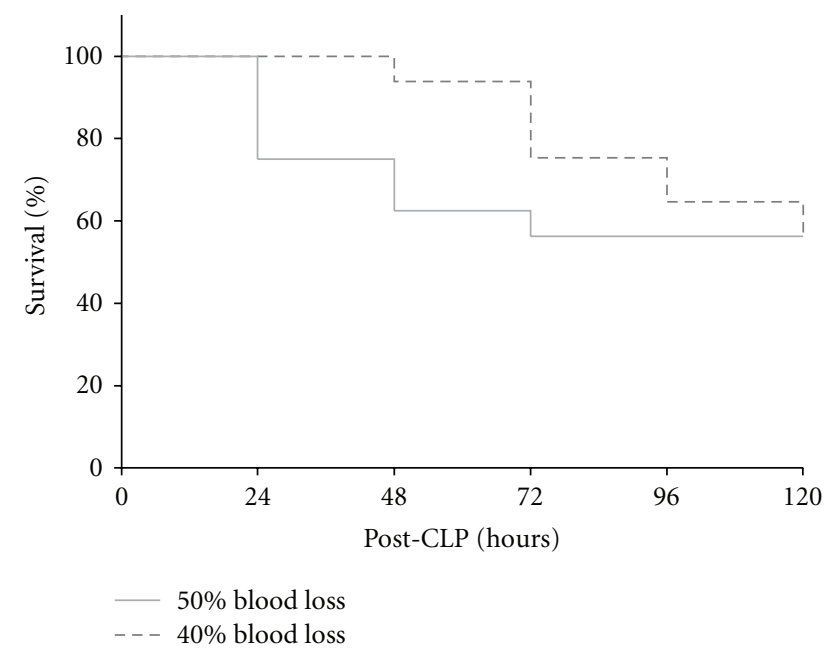

(c)

Figure 2: 7-day mortality in 3-month-old female mice subjected to the two-hit model of trauma/hemorrhage followed by polymicrobial CLP-induced sepsis. To achieve approximately 50\% mortality at post-TH day 7 ( $50 \%$ hemorrhage of total blood volume, TBV), the severity of CLP was adjusted by different gauge needles (20G $n=10,19 \mathrm{G} n=10,18 \mathrm{G} n=10,17 \mathrm{G} n=22)$. To reach sublethality after hemorrhage, $* P<.05$ between 50 and $40 \%$ blood loss group (of TBV) was tested with a 17 -gauge needle $(40 \%+17 \mathrm{G} n=65 ; 50 \%+17 \mathrm{G} n=16)$.

Given the aim of the experiment, sham surgeries were not performed to reduce the total number of mice in the study.

2.3. Blood Sampling. Beginning immediately prior to hemorrhage ( $-48 \mathrm{~h}$ time-point), $20 \mu \mathrm{L}$ of blood was collected via facial vein puncture from each animal every $24 \mathrm{~h}$ (including an additional sample at $6 \mathrm{~h}$ post-CLP) until post-CLP day 5 as previously described by Weixelbaumer et al. [29] (Figure 1). All samples were immediately diluted $1: 10$ in PBS with EDTA (diluted 1:50). After centrifugation $(1 \mathrm{~g}, 5 \mathrm{~min}$, $22^{\circ} \mathrm{C}$ ), $180 \mu \mathrm{L}$ of plasma was removed and stored at $-80^{\circ} \mathrm{C}$ for further analysis.

2.4. Complete Blood Count. After removing plasma, the remaining blood pellet was resuspended with $180 \mu \mathrm{L}$ CellDyn buffer with EDTA prior to complete blood count (CBC) analysis with a Cell Dyn 3700 counter (Abbott Laboratories, Ill, USA).

2.5. Metabolic and Organ Function Parameters. Urea nitrogen (urea), glucose, lactate dehydrogenase (LDH), alanin transaminase (ALT), and aspartate transaminase (AST) were analyzed in plasma samples with a Cobas c111 reader (Roche, Switzerland).

2.6. Statistical Analysis. Survival curves were plotted using Kaplan-Meier method. Data were tested for normality, and for all further analyses, mice were retrospectively divided into either surviving (SUR, alive by post-CLP day 5) or dying (DIE, died between post-CLP day 1 and 5). Due to the high variability and low $n=3$ at $96 \mathrm{~h}$ (post-CLP day 5 ) in the DIE group, we excluded this time point from all graphs/analyses. Regardless of whether pre-CLP (Figures 3-5) or post-CLP 


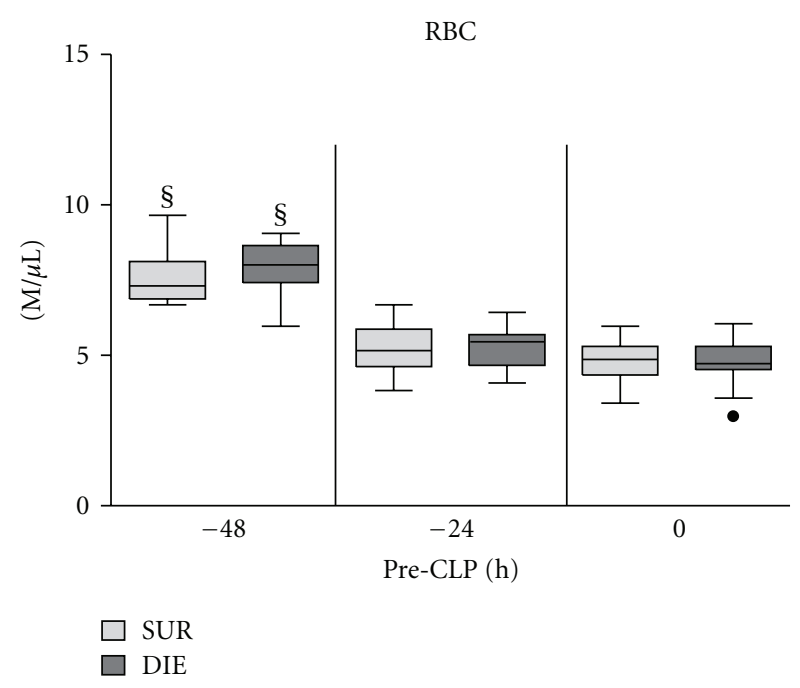

(a)

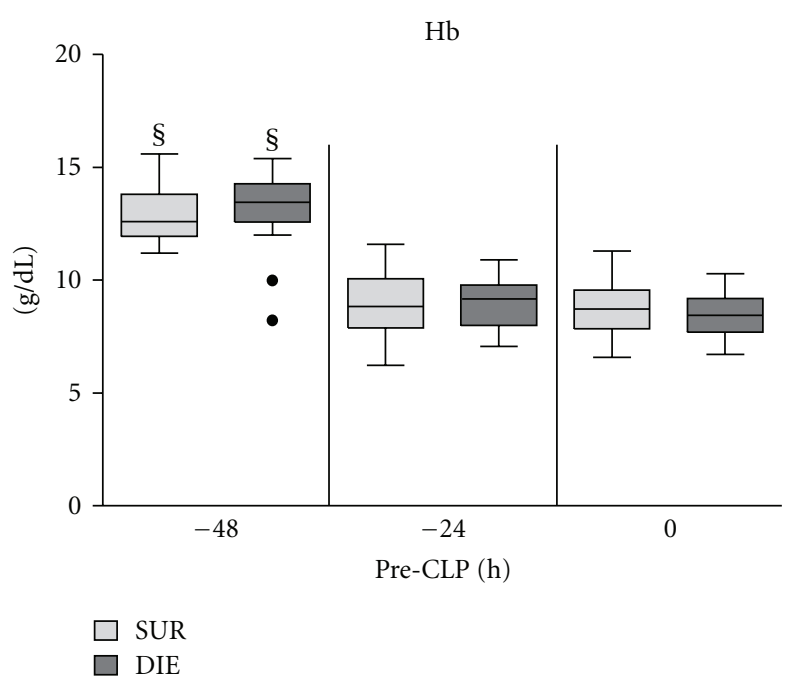

(b)

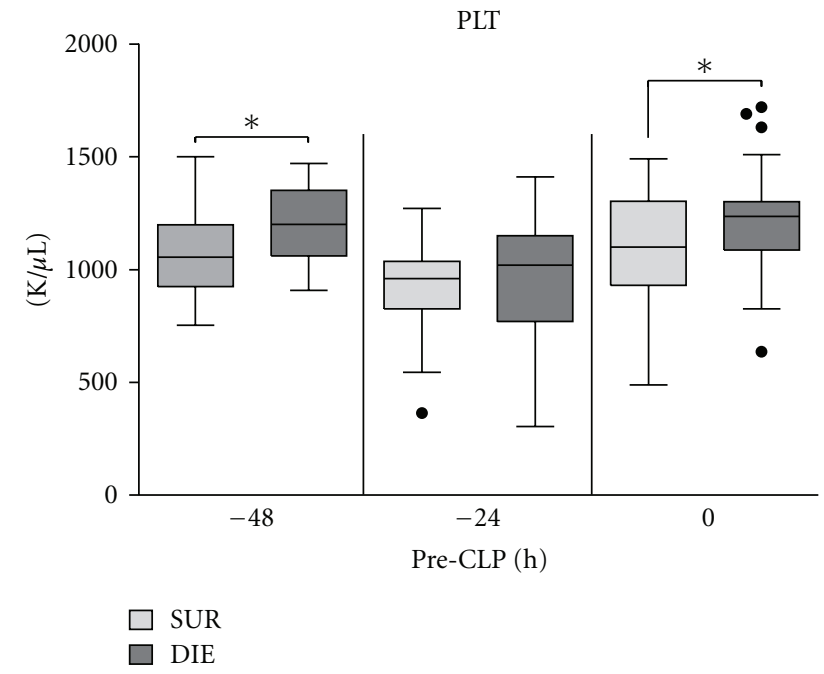

(c)

FIGURE 3: Retrospective comparison of red blood cell, platelet count, and hemoglobin concentration in dying (DIE) versus surviving (SUR) animals prior to CLP. 3-month-old female mice were subjected to trauma/hemorrhage prior to CLP. Displayed values were compared between DIE and SUR at $-48 \mathrm{~h}$ (DIE at least $n=17$, SUR at least $n=18$ ), $-24 \mathrm{~h}$ (DIE at least $n=28$, SUR $n=32$ ), $0 \mathrm{~h}$ before-CLP (DIE at least $n=28$, SUR $n=32$ for all parameters) and between time points. Dots represent outlayers. ${ }^{\S} P<.05$ between time points, and ${ }^{*} P<.05$ between DIE and SUR.

(Figures 6-8), SUR versus DIE differences (each time point separately) were evaluated by either Student's $t$-test (normally distributed data) or Mann-Whitney test (abnormally distributed data). One-way ANOVA followed by Dunn's test was used to assess longitudinal fluctuations of all parameters in the pre-CLP phase. All pre-CLP data (Figures 3-5) are presented as Tukey box-plot diagram, while post-CLP data are plotted as mean \pm SEM (Figures 6-8). Statistical tests were carried out using Prism 5 (GraphPad Software Inc., San Diego, USA), and the level of significance was defined at $P<.05$.

\section{Results}

3.1. Survival. The initial goal was to reach an overall mortality of approximately $50 \%$ at the end of the acute phase of sepsis (post-CLP day 5, post-TH day 7). All mice were subjected to trauma (unilateral femur fracture) and hemorrhage (50\% of TBV) followed by CLP of varying severity $48 \mathrm{~h}$ later (Figure 2(a)). Hemorrhage of $50 \%$ of TBV resulted in an average mortality of $27 \%$ at post-TH day 2 . CLP surgery with $17 \mathrm{G}$ needle produced additional mortality of $34 \%$ leading to the overall mortality of approx. $60 \%$ at post-CLP day 5 (post-TH day 7). CLP with remaining needles was either mildly lethal $(10 \%$ by $19 \mathrm{G})$ or nonlethal (20 and 18G) (Figure 2(a)). To eliminate the excessive loss of mice induced by $\mathrm{TH}$, we reduced the hemorrhage volume to $40 \%$ of TBV. This significantly lowered the post-TH mortality from $27 \%$ to $4 \%$ (Figure 2(b)). All post-TH deaths occurred within $1 \mathrm{~h}$ of the hit.

Next, we tested whether the change in the amount of shed blood (50\% versus $40 \%)$ affected the post-CLP outcome. 


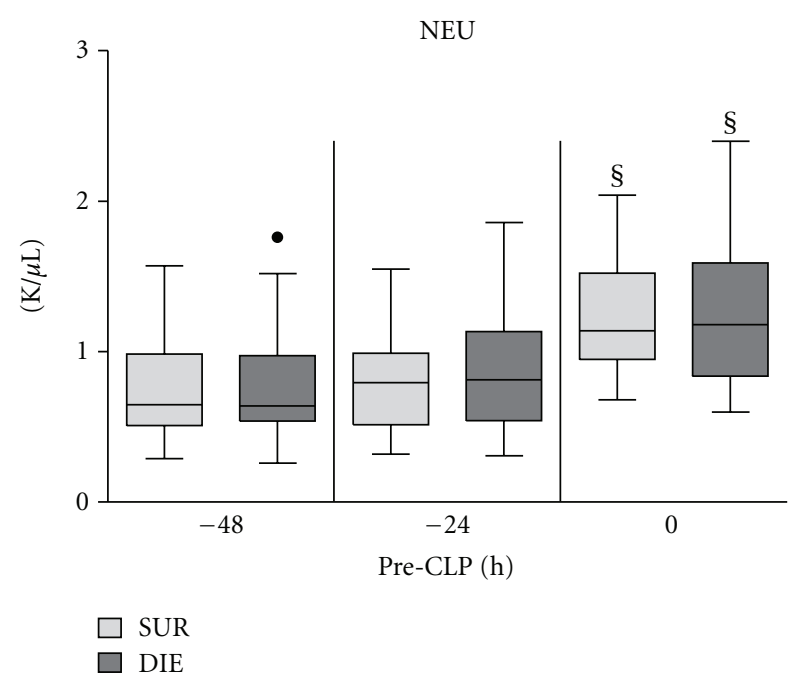

(a)

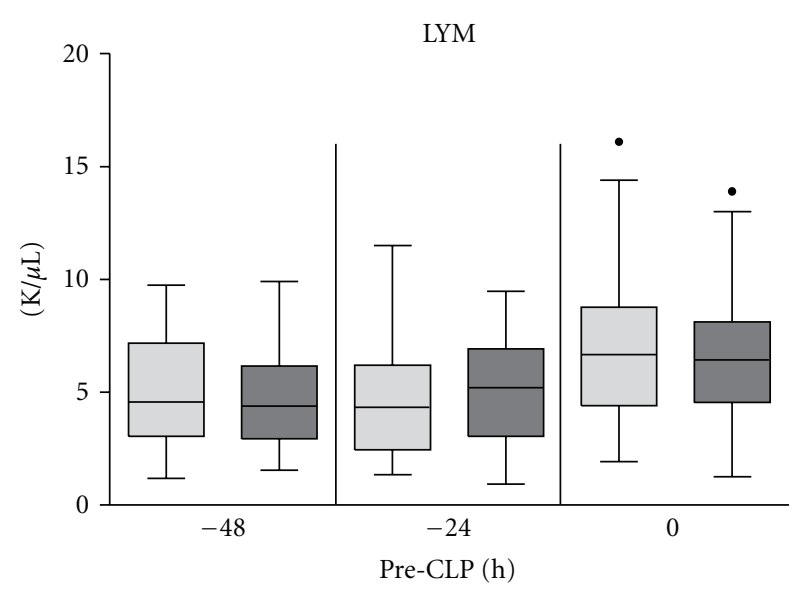

$\square$ SUR

$\square$ DIE

FIGURE 4: Retrospective comparison of neutrophil and lymphocyte count in dying (DIE) versus. surviving (SUR) animals prior to CLP. 3-month-old female mice were subjected to trauma/hemorrhage prior to CLP. Displayed values were compared between DIE and SUR at $-48 \mathrm{~h}$ (DIE $n=17$, SUR $n=18$ ), $-24 \mathrm{~h}$ (DIE $n=29$, SUR $n=32$ ) $0 \mathrm{~h}$ before-CLP (DIE $n=31$, SUR $n=34$ ) and between time points. Dots represent outlayers. ${ }^{\S} P<.05$ between time points.

Mice were subjected to TH with either $50 \%$ or $40 \%$ of TBV followed by CLP with $17 \mathrm{G}$ needle (Figure 2(c)). The (postCLP) day-5 mortality was virtually identical for both groups (56\% versus $54 \%$ ) (Figure $2(\mathrm{c})$ ).

\subsection{Pre-CLP (Post-TH) Phase}

3.2.1. Red Blood Cells, Hemoglobin, and Platelets. In all subsequent experiments, all mice were consistently subjected to TH with $40 \%$ of TBV and CLP with $17 \mathrm{G}$ needle and retrospectively divided into either SUR or DIE for further comparisons (see Section 2.6).

TH hit caused a virtually identical reduction of red blood cell (RBC) counts (by approximately 32\%) and hemoglobin (Hb) concentration (by approx. $31 \%$ ) in both SUR and DIE animals at $-24 \mathrm{~h}$ (Figures 3(a) and 3(b)), and the magnitude of this decrease remained virtually unchanged until CLP $(0 \mathrm{~h}$ time point).

Prior to TH ( $-48 \mathrm{~h}$ time point), platelets (PLT) were lower in SUR by approximately $11 \%(P<.05)$ compared to DIE mice (Figure $3(\mathrm{c})$ ). At $-24 \mathrm{~h}$, PLT insignificantly decreased in SUR (by 13\%) and in DIE (by 20\%) animals (compared to the respective counts at $-48 \mathrm{~h}$ ). At $0 \mathrm{~h}$, PLT recovered to their initial pre-TH values (both groups) with the SUR PLT count approx. $10 \%$ lower $(P<.05)$ than in DIE mice.

3.2.2. White Blood Cells. We investigated the effect of trauma and hemorrhage on neutrophil (NEU) and lymphocyte (LYM) population. TH hit did not affect circulating leukocytes (data not shown). Interestingly, while $\mathrm{TH}$ did not have an immediate effect upon circulating NEU, its delayed surge (identical in both groups) was evident immediately prior to
CLP $(0 \mathrm{~h}$ versus $-48 \mathrm{~h}$ and $-24 \mathrm{~h}, P<.05)($ Figure $4(\mathrm{a}))$. Neither longitudinal nor intergroup differences were observed in post-TH circulating LYM counts (Figure 4(b)).

3.2.3. Organ Function Parameters. TH hit caused a virtually identical hypoglycemia in both SUR and DIE animals, as evidenced by the approx. $40 \%$ drop of circulating glucose at $-24 \mathrm{~h}$ (Figure 5(a)). Glucose remained low (approx. $70 \%$ of the $-48 \mathrm{~h}$ value) in both (SUR and DIE) groups until CLP $(0 \mathrm{~h})$.

There was a strong AST spike in all mice at $-24 \mathrm{~h}$ (Figure 5(b)), and this increase was 3-fold higher in DIE compared to SUR mice $(192.7$ versus $578.7 \mathrm{U} / \mathrm{L}, P<.05)$. However, there was no significant intergroup difference at $0 \mathrm{~h}$, as AST nearly recovered both in SUR $(122 \mathrm{U} / \mathrm{L})$ and DIE (172 U/L) mice (Figure 5(b)). In ALT, there was also a significant post-TH increase at $-24 \mathrm{~h}$, but in contrast to AST, its magnitude (average 48\%) was similar in both SUR and DIE mice (Figure 5(c)).

The same was true for LDH: an initial $64 \%$ post-TH surge at $-24 \mathrm{~h}$ (identical in SUR and DIE) was followed by its marked recovery at $0 \mathrm{~h}$ (Figure 5(d)). Levels of urea differed slightly $(12 \%, P<.05)$ between DIE and SUR prior to $\mathrm{TH}(-48 \mathrm{~h}$, Figure $5(\mathrm{e}))$, but this dissimilarity disappeared at later (post-TH) time points $(-24 \mathrm{~h}$ and $0 \mathrm{~h})$.

\subsection{Post-CLP Phase}

3.3.1. Red Blood Cells, Hemoglobin, and Platelets. Regardless of outcome, RBC counts and $\mathrm{Hb}$ concentration remained decreased throughout the entire post-CLP period. In DIE mice, $\mathrm{RBC}$ and $\mathrm{Hb}$ were slightly (but consistently) elevated (e.g., $15 \%$ and $13 \%$ at $6 \mathrm{~h}$ ) compared to SUR. Sepsis caused an almost identical PLT drop in both SUR and DIE groups 


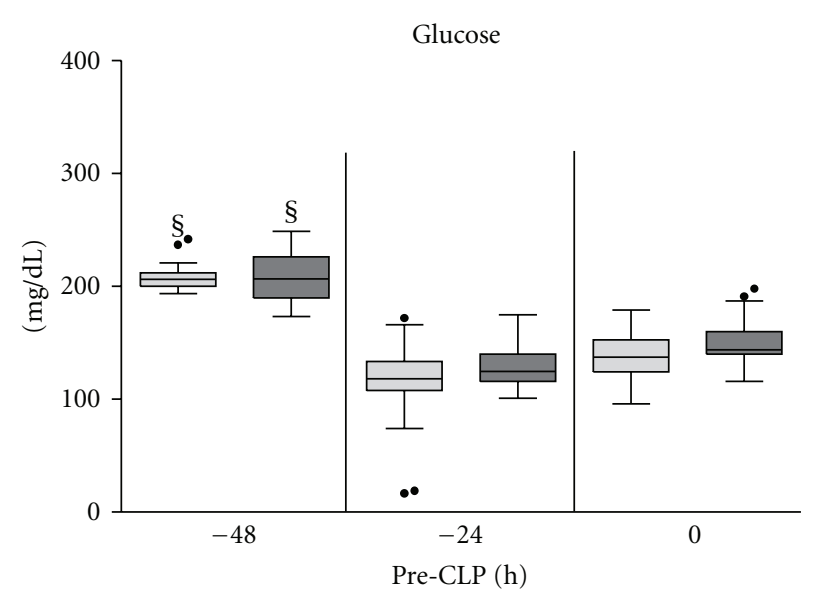

(a)

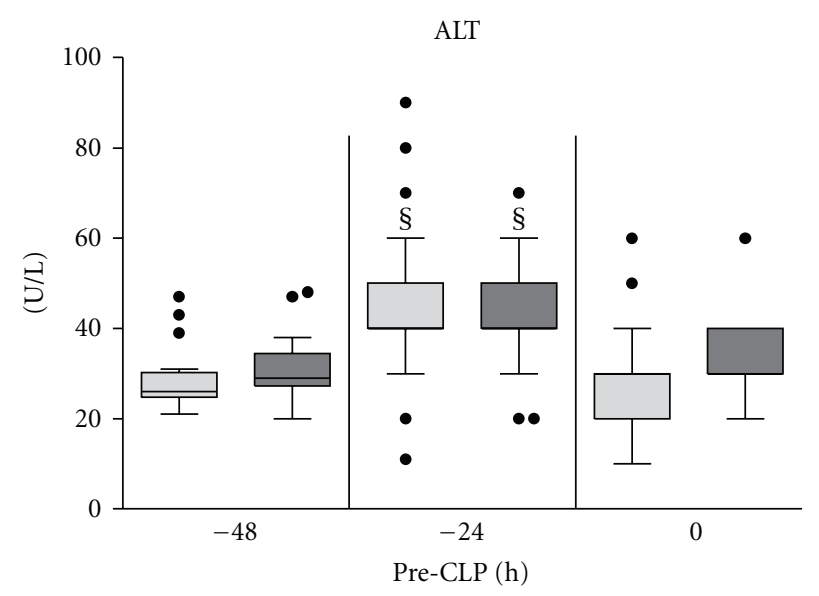

(c)

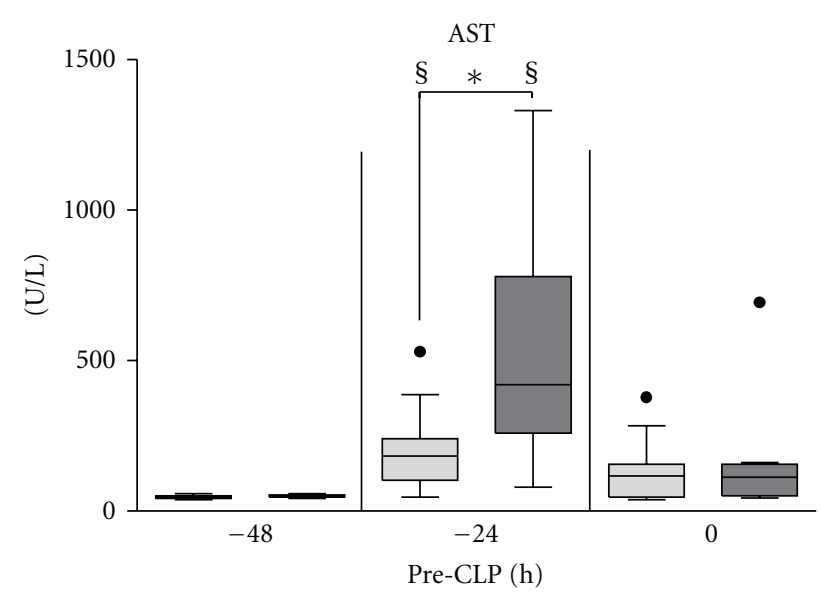

(b)

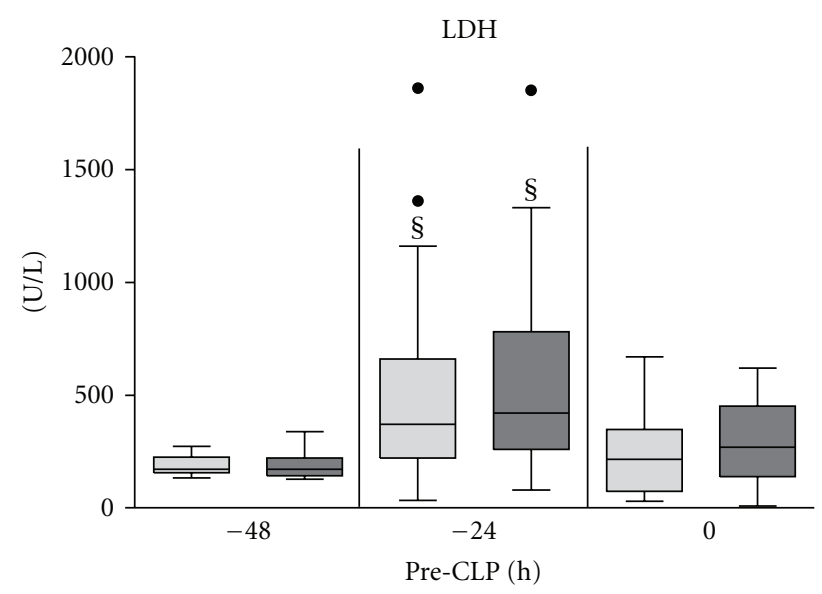

(d)

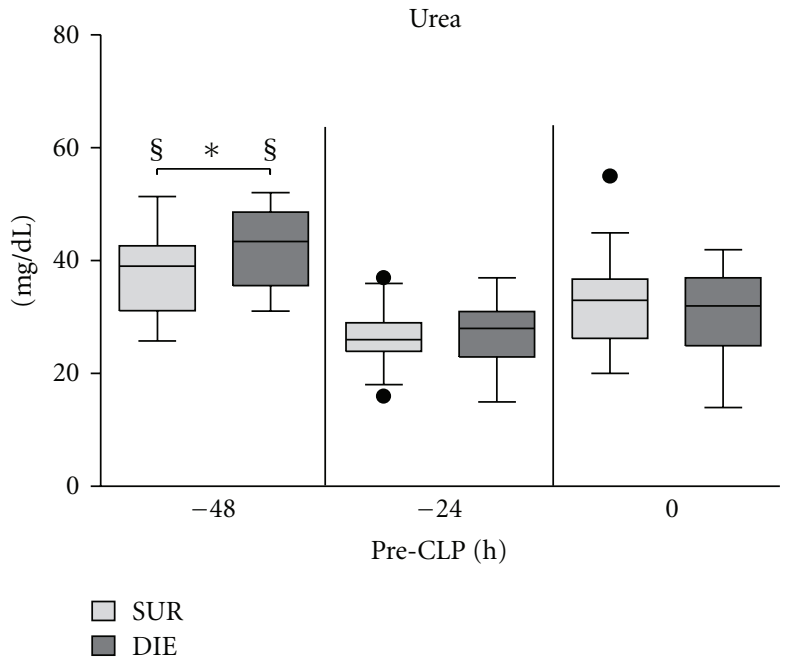

(e)

FIGURE 5: Retrospective comparison of circulating glucose, AST, ALT, LDH, and urea in dying (DIE) versus surviving (SUR) animals prior to CLP. 3-month-old female mice were subjected to trauma/hemorrhage prior to CLP. Displayed values were compared between DIE and SUR at $-48 \mathrm{~h}(\mathrm{DIE} n=16$, SUR $n=18$; AST: DIE $n=6$, SUR $n=12),-24 \mathrm{~h}(\mathrm{DIE} n=23$, SUR $n=32$; AST: SUR $n=21), 0 \mathrm{~h}$ before-CLP (DIE $n=23$, SUR $n=32$; AST: DIE $n=8$, SUR $n=21$ ) and between time points. ${ }^{\S} P<.05$ between time points, and ${ }^{*} P<.05$ between DIE and SUR. 


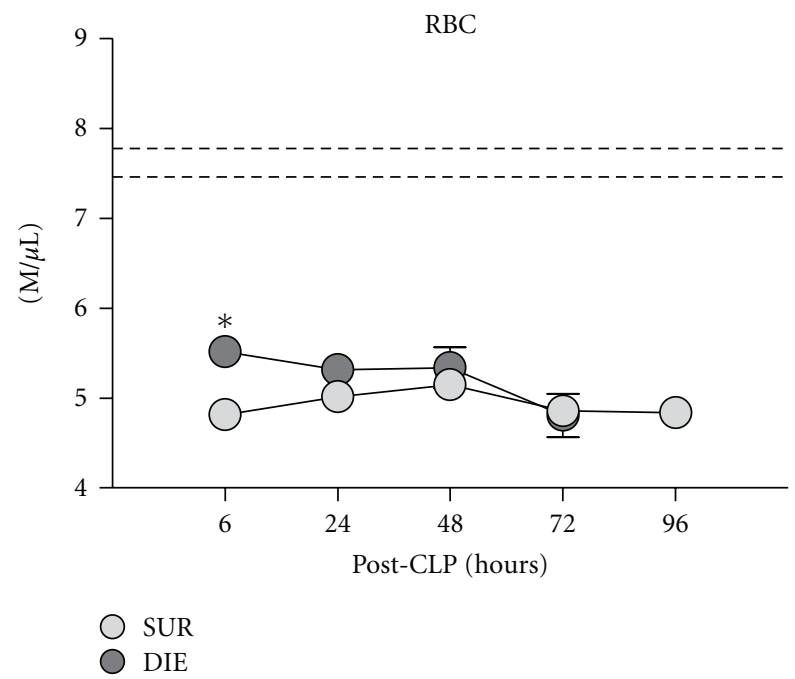

(a)

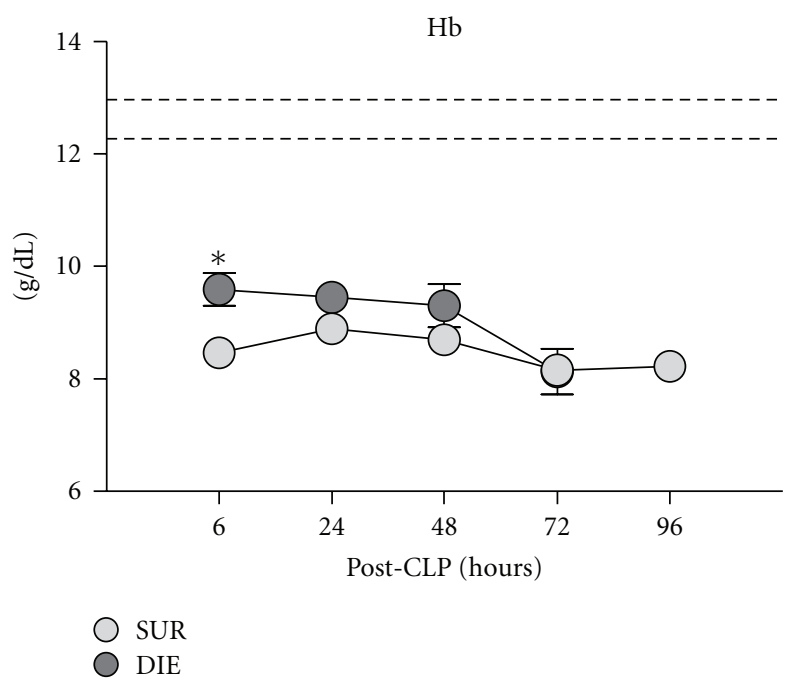

(b)

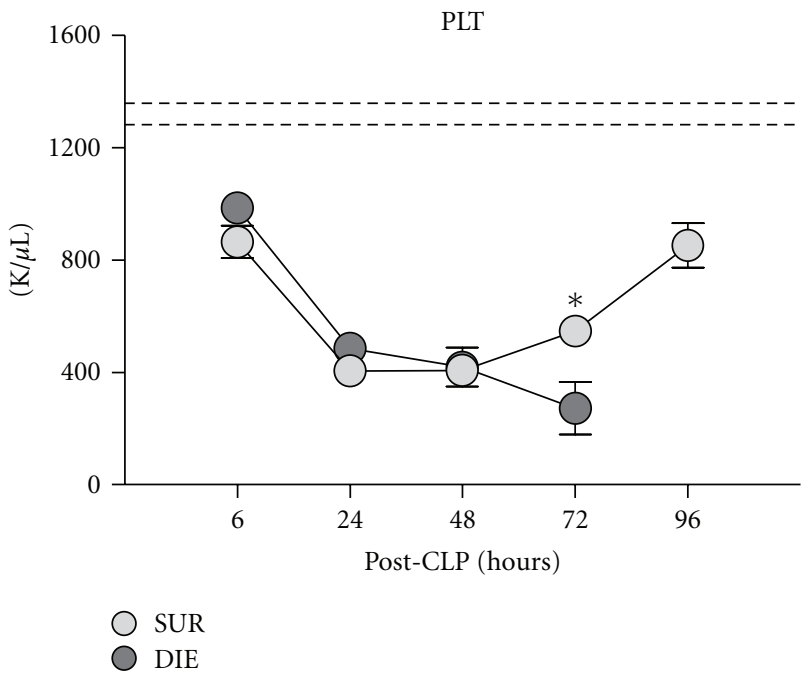

(c)

FIGURE 6: Retrospective comparison of red blood cell platelet count, and hemoglobin concentration in dying (DIE) versus surviving (SUR) animals after-CLP. 3-month-old female mice were subjected to trauma/hemorrhage followed by polymicrobial CLP-induced sepsis. Displayed values were compared between DIE and SUR group $6 \mathrm{~h}$ (DIE at least $n=30$, SUR at least $n=32$ ), $24 \mathrm{~h}$ (DIE at least $n=28$, SUR at least $n=31$ ), $48 \mathrm{~h}$ (DIE at least $n=16$, SUR at least $n=31$ ), $72 \mathrm{~h}$ (DIE at least $n=11$, SUR at least $n=32$ ), and $96 \mathrm{~h}$ (SUR at least $n=26$ for all parameters) after-CLP. Dotted lines represent normal values. ${ }^{*} P<.05$.

until $48 \mathrm{~h}$ post-CLP. Thereafter, PLTs began to recover in SUR mice (further decline in DIE), reaching approx $80 \%$ of baseline at $96 \mathrm{~h}$. A 2-fold separation between SUR and DIE mice was recorded at $72 \mathrm{~h}$ (Figure 6(c)).

3.3.2. White Blood Cells. Within the first $48 \mathrm{~h}$, CLP resulted in a drop of NEU in all mice with NEU counts typically lower (e.g., by $31 \%$ at $24 \mathrm{~h}$ and $21 \%$ at $72 \mathrm{~h}$ ) in DIE animals compared to SUR (Figure $7(\mathrm{a})$ ). From $48 \mathrm{~h}$ onward, there was a gradual recovery of NEU, which at $72 \mathrm{~h}$ either reached (in DIE) or exceeded (in SUR) baseline values.

Similar to NEU, there was a rapid LYM decrease in all mice within the first $48 \mathrm{~h}$ of CLP, with initial LYM counts significantly higher (by $17 \%$ at $6 \mathrm{~h}$ and $51 \%$ at $24 \mathrm{~h}$ ) in
DIE compared to SUR (Figure 7(b)). This was followed by a prominent shift between the SUR versus DIE groups: while the $72 \mathrm{~h}$ LYM count remained depressed in DIE mice, there was a robust LYM recovery in SUR from $48 \mathrm{~h}$ onward. At $72 \mathrm{~h}$, the SUR LYM count was higher by $40 \%(P<.05)$ (compared to the LYM count in DIE) and continued to increase toward baseline (approx. 84\% of the lower baseline range at $96 \mathrm{~h}$ ) (Figure $7(\mathrm{~b})$ ).

3.3.3. Organ Function Parameters. Regardless of the group, preexisting (post-TH) hypoglycemia worsened after the CLPinduced sepsis. Compared to DIE, a slight glucose recovery in SUR mice was noted from $48 \mathrm{~h}$ onward (by approx. $20 \%$ at $48 \mathrm{~h}$ and $23 \%$ at $72 \mathrm{~h}, P<.05$ ) (Figure $8(\mathrm{a})$ ). In both DIE and 


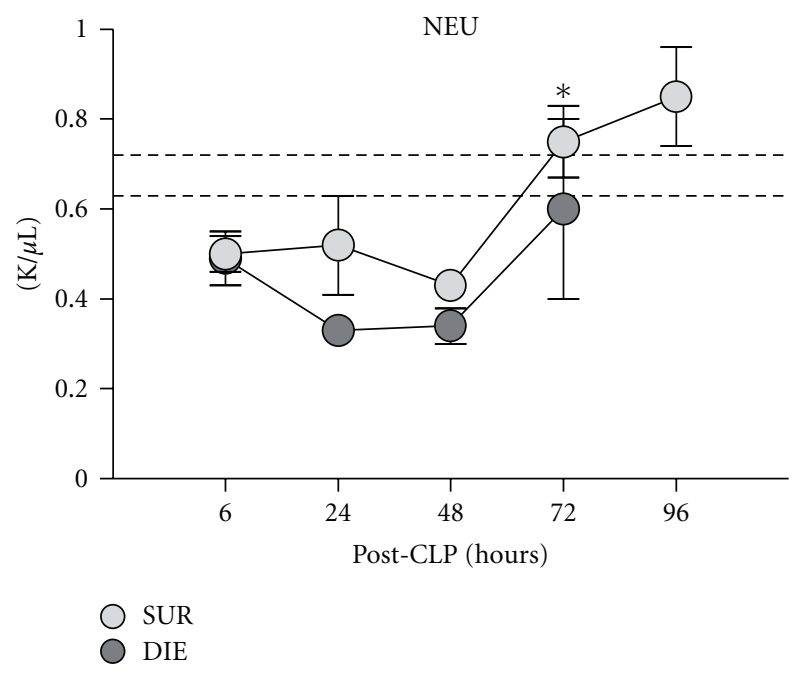

(a)

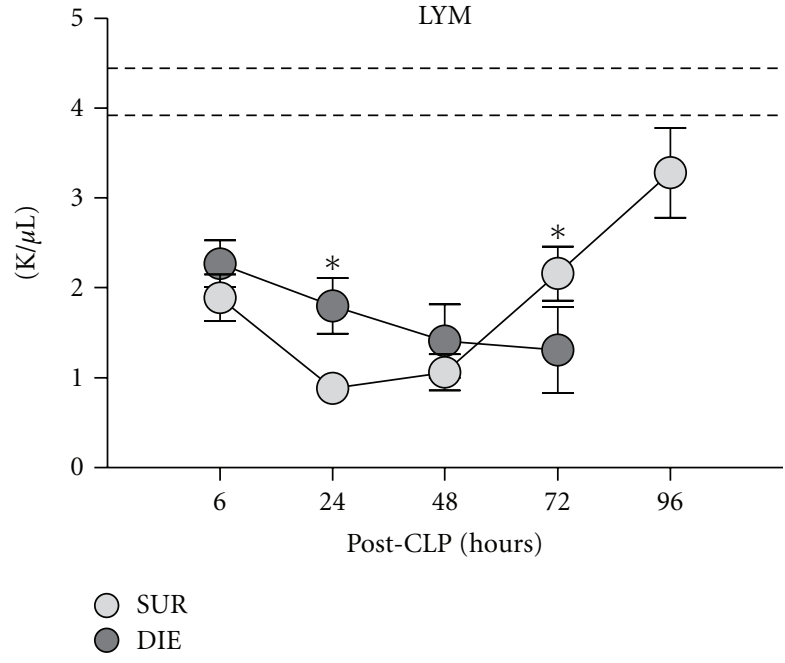

(b)

FIGURE 7: Retrospective comparison of neutrophil and lymphocyte count in dying (DIE) versus surviving (SUR) animals after-CLP. 3month-old female mice were subjected to trauma/hemorrhage followed by polymicrobial CLP-induced sepsis. Displayed values were compared between DIE and SUR groups $6 \mathrm{~h}$ (DIE $n=31$, SUR $n=33$ ), $24 \mathrm{~h}$ (DIE $n=29$, SUR $n=34$ ), 48 h (DIE $n=18$, SUR $n=32), 72 \mathrm{~h}(\mathrm{DIE} n=12$, SUR $n=32)$, and $96 \mathrm{~h}(\operatorname{SUR} n=28)$ after-CLP. Dotted lines represent normal values. ${ }^{*} P<.05$.

SUR groups, there was a notable post-CLP increase in AST. The peak inter-group difference (DIE 2-fold higher than SUR) was recorded at $48 \mathrm{~h}(P<.05)$ but disappeared at $72 \mathrm{~h}$ (Figure $8(\mathrm{~b})$ ). Such an intergroup separation was even more pronounced in ALT: while its activity continuously increased in DIE from $6 \mathrm{~h}$ onward, it was gradually declining (transient peak at $24 \mathrm{~h}$ ) in SUR reaching baseline by $96 \mathrm{~h}$ (Figure 8(c)). The maximal, 6-fold difference in circulating ALT between SUR and DIE mice was recorded at $72 \mathrm{~h}(0.3$-fold at $24 \mathrm{~h}$ and 4 -fold at $48 \mathrm{~h}$ ).

Similar after-CLP response was observed for LDH and urea. Both parameters were elevated in DIE mice: urea by 0.3 -fold at $6 \mathrm{~h}$ and by 3.6 -fold between $24-72 \mathrm{~h}$, while LDH by approximately 2 -fold at $24 \mathrm{~h}, 5$-fold at $48 \mathrm{~h}$, and 3.5 -fold at $72 \mathrm{~h}$ time point $(P<.05)$ compared to SUR. In SUR mice, both urea and LDH values approached baseline from $48 \mathrm{~h}$ onward.

\section{Discussion}

The primary goal of our study was to reproduce a scenario, in which an initial traumatic/hypovolemic event survived by an ICU-monitored patient is complicated by severe polymicrobial sepsis. By combining the two-hit model with daily blood sampling, we were able to detect potential early and late differences between surviving and dying septic animals and follow the evolution of those changes prior and/or after each of the hits.

Given that an extensive blood loss typically coincides with injury and/or trauma, combining these two elements into an initial challenge is more clinically relevant than referring to established first-hit models consisting solely of hemorrhage [30]. Elimination of either trauma or hemorrhage component may significantly distort the ensuing pathophysiological immunoinflammatory response, and in effect produce incomplete or erroneous conclusions. The growing awareness to the above-mentioned facts has provoked attempts to develop more representative (two-hit) animal models aimed at investigating the pathogenesis of sepsis as the secondary insult.

Nosocomial (ventilator-associated) pneumonia is a major cause of secondary septic complications in trauma patients with prolonged ICU stays $[31,32]$ although incidences of polymicrobial sepsis are also reported [33]. An abdominal focus of infection may be viewed as relatively suboptimal given the prevalence of lung-associated secondary infections in polytrauma patients. In our model, however, the cecal ligation and puncture primarily served as a trigger to produce a severe polymicrobial challenge (as opposed to the typically monobacterial lung-associated sepsis) that spreads systemically in a protracted fashion, rather than a model of abdominal peritonitis per se. Moreover, our model was deliberately deprived of relevant intangibles such as preexisting comorbidities (e.g., diabetes, atherosclerosis) and operative interventions that are routinely observed/performed in patients and may significantly skew investigated parameters and outcome $[34,35]$. We chose to use healthy mice since any confounding factor in addition to the two-hit challenge would likely generate too much variability and make data interpretation difficult. The CLP challenge itself provides an adequate variability regarding both the outcome and wide range of generated responses. The level of sepsis-induced mortality in our model (approx. $50 \%$ at day 5 post-CLP) was carefully set to closely reproduce clinical data (reporting between $20 \%$ and $60 \%$ incidence of deaths in the septic ICU patients) [12]. This is important since data generated in highly lethal CLP models might artificially enhance predictive value of 


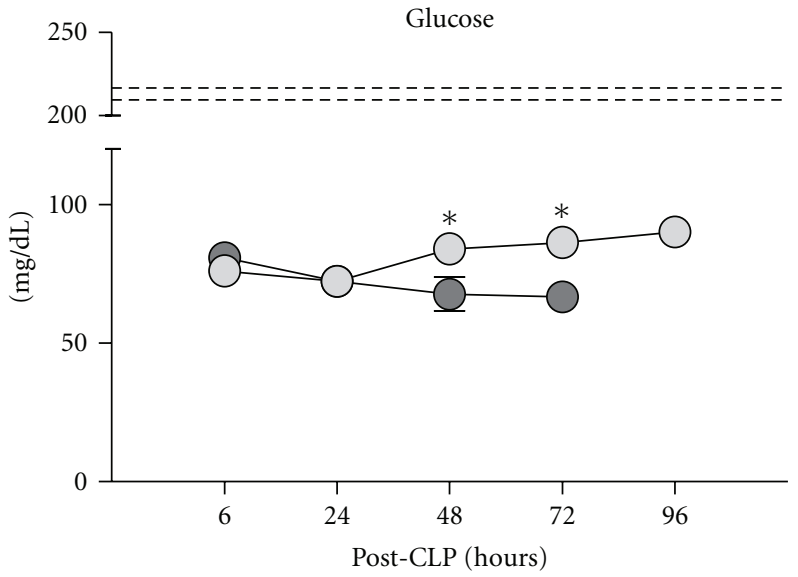

(a)

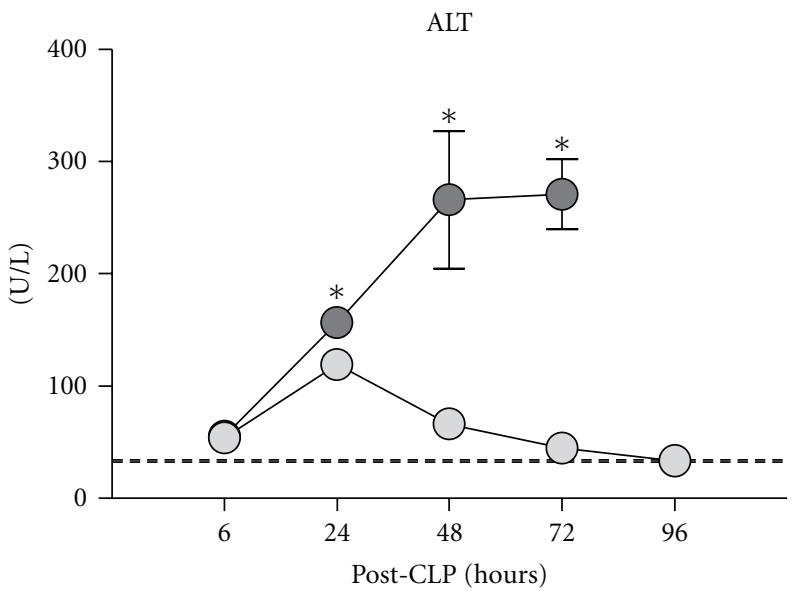

(c)

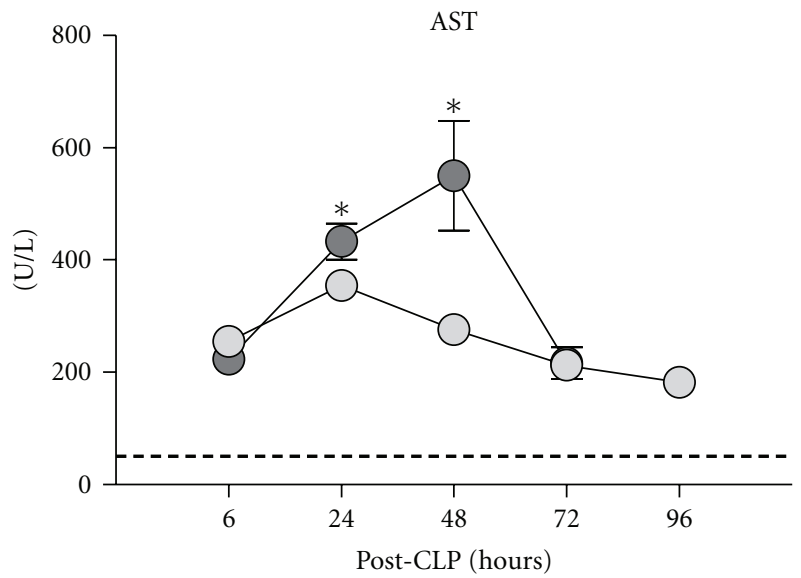

(b)

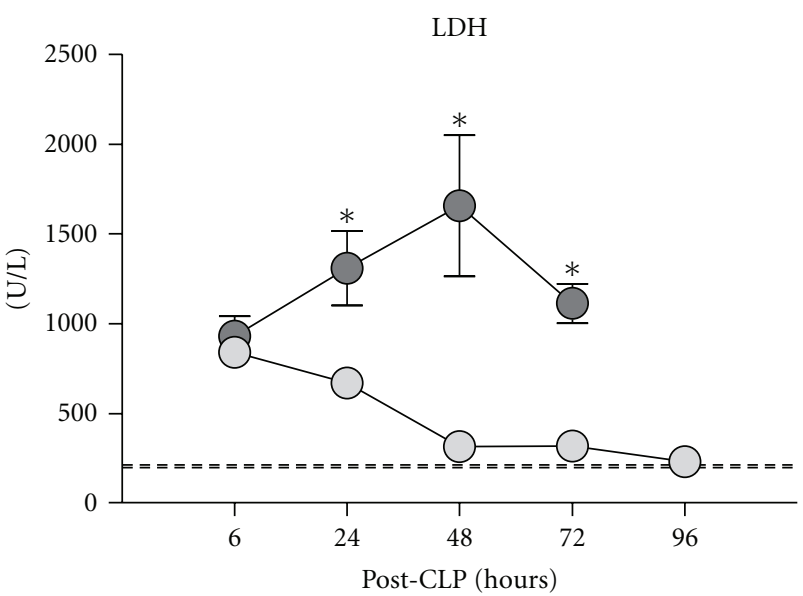

(d)

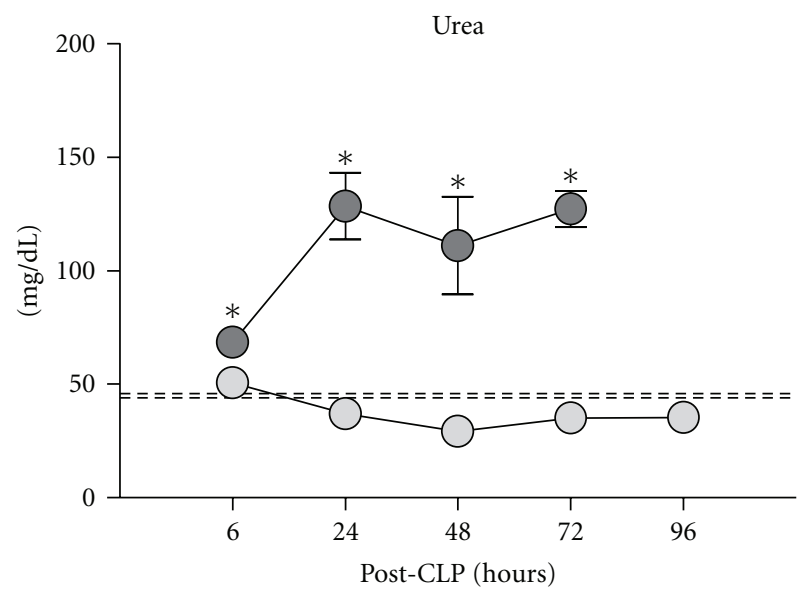

SUR

DIE

(e)

FIGURE 8: Retrospective comparison of circulating glucose, AST, ALT, LDH, and urea in dying (DIE) versus surviving (SUR) animals afterCLP. 3-month-old female mice were subjected to trauma/hemorrhage followed by polymicrobial CLP-induced sepsis. Displayed values were compared between DIE and SUR groups $6 \mathrm{~h}$ (DIE $n=23$, SUR $n=32$; AST: DIE $n=8$, SUR $n=20), 24 \mathrm{~h}$ (DIE $n=28$, SUR $n=34$; AST: DIE $n=14$, SUR $n=23), 48 \mathrm{~h}($ DIE $n=19$, SUR $n=34$; AST: DIE $n=9$, SUR $n=23), 72 \mathrm{~h}(\operatorname{DIE} n=12$, SUR $n=34$; AST: DIE $n=3$, SUR $n=23$ ), and $96 \mathrm{~h}$ (SUR at least $n=33$; AST: SUR $n=23$ ) after-CLP. Dotted lines represent normal values. ${ }^{*} P<.05$. 
some biomarkers [36]. For example, we noted a significant improvement in predictive accuracy for outcome of IL-6 in severe $\left(\mathrm{LD}_{80}\right)$ CLP model compared to a milder $\left(\mathrm{LD}_{30}\right)$ CLP injury (unpublished observation).

Both hemorrhage and trauma elements of the first hit were modified from our original protocol [19]: guillotine was replaced with custom-built blunt pliers while the hemorrhage step was initiated immediately after femur fracture (versus a $2 \mathrm{~h}$ delay in the previous setup), and the hemorrhage volume was decreased by $20 \%$ (to $40 \%$ of TBV). In our laboratory, the use of pliers better reproduced blunt soft tissue damage while still providing an excellent reproducibility of noncomminuted, midshaft femur fracture. The hemorrhage modifications were dictated by both the desire to reproduce a more clinically relevant timing and to produce a survivable hypovolemic shock. Given that in trauma patients fractures naturally diverge in terms of location, type and severity of concurrent soft tissue injury and extensive hemorrhage is frequently lethal, a relatively rigorous standardization of these elements can be viewed as the model's shortcoming rather than advantage. Yet, in this particular two-hit setup, a relatively similar magnitude of the $\mathrm{TH}$ hit severity and maximal elimination of lethality are desired, as we aimed to verify our model by studying the outcome-dependent responses triggered by the CLP hit (and not by the TH). Additionally, extrapolability of potential responses indicative of the post-CLP outcome is more relevant if such diverse reactions were produced by a conservatively standardized (hence generating more uniform responses) TH hit. Finally, the adherence to the RussellBurch " $3 \mathrm{R}$ " tenet (replace, refine, reduce) should not be overlooked [37].

Profound changes in the immunoinflammatory status caused by trauma/hemorrhage, specifically those propagating development of progressive immunoparalysis, have been postulated to be responsible for the increased susceptibility to secondary sepsis [36]. Although, due to technical constraints, we were not able to determine the cytokine-based immunoinflammatory status of TH-CLP mice, this aspect requires careful evaluation in subsequent experiments. Given that immuno-inflammatory fluctuations frequently occur in patients over the period of several hours, a rigorous and continuous surveillance of the ICU patients' immune status is fundamental in ensuring the most adequate therapeutic strategy (e.g., rapid adjustments of treatment to tailor it to the ongoing shifts of the immune system). To enable such tactics in our TH-CLP model, we subjected all mice to daily low-volume blood sampling via facial vein puncture. This technique provides daily collection of blood ( $20 \mu \mathrm{L} /$ mouse) from critically ill mice with virtually no negative effects upon their outcome and/or health status [29]. Despite a limited sample volume available for analysis, advanced and highly sensitive techniques such as multiplex bead assays and various automated biochemistry analyzers allow accurate measurement of dozens of circulating biomarkers and/or cell subpopulations. However, in the context of a joint critical disease/repetitive sampling protocol, its main advantage lies in an ability to investigate a natural evolution of a given disease: any relevant immuno-inflammatory, metabolic, and/or organ function alterations can be followed over time in individual subjects to an undefined outcome. This is in stark contrast to studies in which animals are sacrificed at predefined time points providing only a narrow snapshot assessment of investigated endpoints/pathways without option to match recorded changes with outcome.

In our study, we employed the TH-CLP/monitoring combination to gain preliminary insight into outcomerelated mechanisms developing during the acute phase of post-traumatic sepsis. In context of potential posttraumatic septic complications, the period between the first (trauma/hemorrhage) and the secondary (sepsis) challenge may be as decisive as the immediate time span following the onset of sepsis. If relevant risk-correlating marker(s) are identified in the posttraumatic but preseptic phase, one could identify not only subjects with high risk of developing septic complications (posttrauma) but also those who display higher risk of death once sepsis sets in. While this model does not allow any insight into the first scenario (all mice undergo sepsis), it is well matched to investigate the latter situation. We therefore performed outcome-based retrospective analysis of all analysed parameters to detect potential early (pre-CLP) and late (post-CLP) differences between surviving and dying individuals. In the pre-CLP phase, there was a much stronger post-TH $(24 \mathrm{~h}$ pre-CLP) AST spike (indirectly indicative of hepatic injury) in mice that died within first few days after the onset of peritonitis (compared to survivors), whereas the same cohort (DIE) showed clear and profound metabolic/organ function disturbances in the post-CLP period. Further detailed analysis are needed to establish whether some post-TH mice develop a more intense dysfunction of selected organs prior to sepsis (consequently predisposing them for unfavourable post-CLP outcome) and how exactly organ disturbances drive lethal septic responses both prior and after the onset of sepsis. In any respect, such longitudinal assessments, enabled by more ICU-like tailored models such as the one described above, generate valuable findings that may be then successfully extrapolated to respective clinical scenarios. This in turn may help in identifying ideal time points and/or targets for successful therapeutic interventions in sepsis and other related critical diseases.

\section{Conclusion}

The modified and clinically relevant TH-CLP model allows a more adequate investigative insight into the course of (polymicrobial) septic complications following trauma. Additional integration of the daily blood sampling into the TH-CLP setup closely emulates one of the major components of the ICU protocol: repetitive monitoring of critically sick patients. The daily monitoring of $\mathrm{TH}$ CLP mice was a basis for outcome-based (retrospective) stratification of responses they generated during both twohit phases. Such an approach enables in turn correlation of circulating cells/biomarkers to septic outcomes even prior to the development of sepsis. If verified in a clinical setting, findings from such advanced models not only could help 
to categorize patients into high-and low-risk of death after their traumatic insult gets complicated by sepsis, but also to prevent the onset of septic sequelae altogether.

\section{Acknowledgments}

The study was supported by the Vienna Science and Technology Fund (WWTF Grant no. LS07-065). The authors gratefully acknowledge the expert help of Anna Khadem and Christine Kober with the technically challenging organ function assays.

\section{References}

[1] D. C. Angus, W. T. Linde-Zwirble, J. Lidicker, G. Clermont, J. Carcillo, and M. R. Pinsky, "Epidemiology of severe sepsis in the United States: analysis of incidence, outcome, and associated costs of care," Critical Care Medicine, vol. 29, no. 7, pp. 1303-1310, 2001.

[2] J. L. Vincent, "EPIC II: sepsis around the world," Minerva Anestesiologica, vol. 74, no. 6, pp. 293-296, 2008.

[3] E. Christaki and S. M. Opal, "Is the mortality rate for septic shock really decreasing?" Current Opinion in Critical Care, vol. 14 , no. 5, pp. 580-586, 2008.

[4] D. J. Stearns-Kurosawa, M. F. Osuchowski, C. Valentine, S. Kurosawa, and D. G. Remick, "The pathogenesis of sepsis," Annual Review of Pathology, vol. 6, 2011.

[5] R. S. Hotchkiss, C. M. Coopersmith, J. E. McDunn, and T. A. Ferguson, "The sepsis seesaw: tilting toward immunosuppression," Nature Medicine, vol. 15, no. 5, pp. 496-497, 2009.

[6] M. F. Osuchowski, K. Welch, J. Siddiqui, and D. G. Remick, "Circulating cytokine/inhibitor profiles reshape the understanding of the SIRS/CARS continuum in sepsis and predict mortality," Journal of Immunology, vol. 177, no. 3, pp. 19671974, 2006.

[7] J. L. Vincent, Y. Sakr, C. L. Sprung et al., "Sepsis in European intensive care units: results of the SOAP study," Critical Care Medicine, vol. 34, no. 2, pp. 344-353, 2006.

[8] D. Nast-Kolb, M. Aufmkolk, S. Rucholtz, U. Obertacke, and C. Waydhas, "Multiple organ failure still a major cause of morbidity but not mortality in blunt multiple trauma," Journal of Trauma, vol. 51, no. 5, pp. 835-842, 2001.

[9] S. Shalhub, C. E. Junker, S. D. Imahara, M. N. Mindrinos, S. Dissanaike, and G. E. O'keefe, "Variation in the TLR4 gene influences the risk of organ failure and shock posttrauma: a cohort study," Journal of Trauma, vol. 66, no. 1, pp. 115-122, 2009.

[10] O. Brattstrom, F. Granath, P. Rossi, and A. Oldner, "Early predictors of morbidity and mortality in trauma patients treated in the intensive care unit," Acta Anaesthesiologica Scandinavica, vol. 54, no. 8, pp. 1007-1017, 2010.

[11] T. M. Osborn, J. K. Tracy, J. R. Dunne, M. Pasquale, and L. M. Napolitano, "Epidemiology of sepsis in patients with traumatic injury," Critical Care Medicine, vol. 32, no. 11, pp. 2234-2240, 2004.

[12] L. F. Poli-de-Figueiredo, A. G. Garrido, N. Nakagawa, and P. Sannomiya, "Experimental models of sepsis and their clinical relevance," Shock, vol. 30, no. 1, pp. 53-59, 2008.

[13] H. Redl and S. Bahrami, "Large animal models: baboons for trauma, shock, and sepsis studies," Shock, vol. 24, no. 1, pp. 88-93, 2005.
[14] D. G. Remick and P. A. Ward, "Evaluation of endotoxin models for the study of sepsis," Shock, vol. 24, no. 1, pp. 7-11, 2005.

[15] C. T. Esmon, "Why do animal models (sometimes) fail to mimic human sepsis?" Critical Care Medicine, vol. 32, no. 5, pp. S219-222, 2004.

[16] W. J. Hubbard, M. Choudhry, M. G. Schwacha et al., "Cecal ligation and puncture," Shock, vol. 24, no. 1, pp. 52-57, 2005.

[17] N. Zantl, A. Uebe, B. Neumann et al., "Essential role of gamma interferon in survival of colon ascendens stent peritonitis, a novel murine model of abdominal sepsis," Infection and Immunity, vol. 66, no. 5, pp. 2300-2309, 1998.

[18] R. N. Stephan, T. S. Kupper, and A. S. Geha, "Hemorrhage without tissue trauma produces immunosuppression and enhances susceptibility to sepsis," Archives of Surgery, vol. 122, no. 1, pp. 62-68, 1987.

[19] M. Van Griensven, M. Kuzu, M. Breddin et al., "Polymicrobial sepsis induces organ changes due to granulocyte adhesion in a murine two hit model of trauma," Experimental and Toxicologic Pathology, vol. 54, no. 3, pp. 203-209, 2002.

[20] M. L. Steinhauser, C. M. Hogaboam, S. L. Kunkel, N. W. Lukacs, R. M. Strieter, and T. J. Standiford, "IL-10 is a major mediator of sepsis-induced impairment in lung antibacterial host defense," Journal of Immunology, vol. 162, no. 1, pp. 392399, 1999.

[21] E. D. Murphey, C. Y. Lin, R. W. McGuire, T. Toliver-Kinsky, D. N. Herndon, and E. R. Sherwood, "Diminished bacterial clearance is associated with decreased IL-12 and interferongamma production but a sustained proinflammatory response in a murine model of postseptic immunosuppression," Shock, vol. 21, no. 5, pp. 415-425, 2004.

[22] J. T. Muenzer, C. G. Davis, B. S. Dunne, J. Unsinger, W. M. Dunne, and R. S. Hotchkiss, "Pneumonia after cecal ligation and puncture: a clinically relevant "two-hit" model of sepsis," Shock, vol. 26, no. 6, pp. 565-570, 2006.

[23] W. J. Kox, T. Volk, S. N. Kox, and H. D. Volk, "Immunomodulatory therapies in sepsis," Intensive Care Medicine, vol. 26, supplement 1, pp. S124-S128, 2000.

[24] M. F. Osuchowski, J. Connett, K. Welch, J. Granger, and D. G. Remick, "Stratification is the key: inflammatory biomarkers accurately direct immunomodulatory therapy in experimental sepsis," Critical Care Medicine, vol. 37, no. 5, pp. 1567-1573, 2009.

[25] K. A. Wichterman, A. E. Baue, and I. H. Chaudry, "Sepsis and septic shock: a review of laboratory models and a proposal," Journal of Surgical Research, vol. 29, no. 2, pp. 189-201, 1980.

[26] I. R. Turnbull, P. Javadi, T. G. Buchman, R. S. Hotchkiss, I. E. Karl, and C. M. Coopersmith, "Antibiotics improve survival in sepsis independent of injury severity but do not change mortality in mice with markedly elevated interleukin 6 levels," Shock, vol. 21, no. 2, pp. 121-125, 2004.

[27] S. L. Zanotti-Cavazzoni, M. Guglielmi, J. E. Parrillo, T. Walker, R. P. Dellinger, and S. M. Hollenberg, "Fluid resuscitation influences cardiovascular performance and mortality in a murine model of sepsis," Intensive Care Medicine, vol. 35, no. 4, pp. 748-754, 2009.

[28] R. P. Dellinger, M. M. Levy, J. M. Carlet et al., "Surviving sepsis campaign: international guidelines for management of severe sepsis and septic shock: 2008," Critical Care Medicine, vol. 36, no. 1, pp. 296-327, 2008.

[29] K. M. Weixelbaumer, P. Raeven, H. Redl, M. van Griensven, S. Bahrami, and M. F. Osuchowski, "Repetitive, low-volume blood sampling method as a feasible monitoring tool in a 
mouse model of sepsis," Shock, vol. 34, no. 4, pp. 420-426, 2010.

[30] R. A. Catania and I. H. Chaudry, "Immunological consequences of trauma and shock," Annals of the Academy of Medicine Singapore, vol. 28, no. 1, pp. 120-132, 1999.

[31] A. Cook, S. Norwood, and J. Berne, "Ventilator-associated pneumonia is more common and of less consequence in trauma patients compared with other critically ill patients," Journal of Trauma, vol. 69, no. 5, pp. 1083-1091, 2010.

[32] M. Magret, R. Amaya-Villar, J. Garnacho et al., "Ventilatorassociated pneumonia in trauma patients is associated with lower mortality: results from EU-VAP study," Journal of Trauma, vol. 69, no. 4, pp. 849-854, 2010.

[33] A. Cheron, B. Floccard, B. Allaouchiche et al., "Lack of recovery in monocyte human leukocyte antigen-DR expression is independently associated with the development of sepsis after major trauma," Critical Care, vol. 14, no. 6, p. R208, 2010.

[34] H. Xiao, J. Siddiqui, and D. G. Remick, "Mechanisms of mortality in early and late sepsis," Infection and Immunity, vol. 74, no. 9, pp. 5227-5235, 2006.

[35] M. F. Osuchowski, F. L. Craciun, E. Schuller, C. Sima, R. Gyurko, and D. G. Remick, "Untreated type 1 diabetes increases sepsis-induced mortality without inducing a prelethal cytokine response," Shock, vol. 34, no. 4, pp. 369-376, 2010.

[36] M. J. Delano and L. L. Moldawer, "Magic bullets and surrogate biomarkers circa 2009," Critical Care Medicine, vol. 37, no. 5, pp. 1796-1798, 2009.

[37] P. Hobson-West, "What kind of animal is the "Three Rs"?" Alternatives to Laboratory Animals, vol. 37, no. 2, pp. 95-99, 2009. 


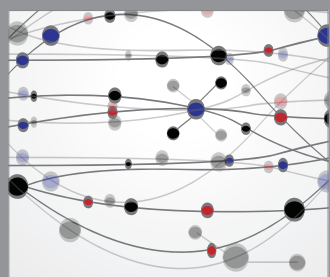

The Scientific World Journal
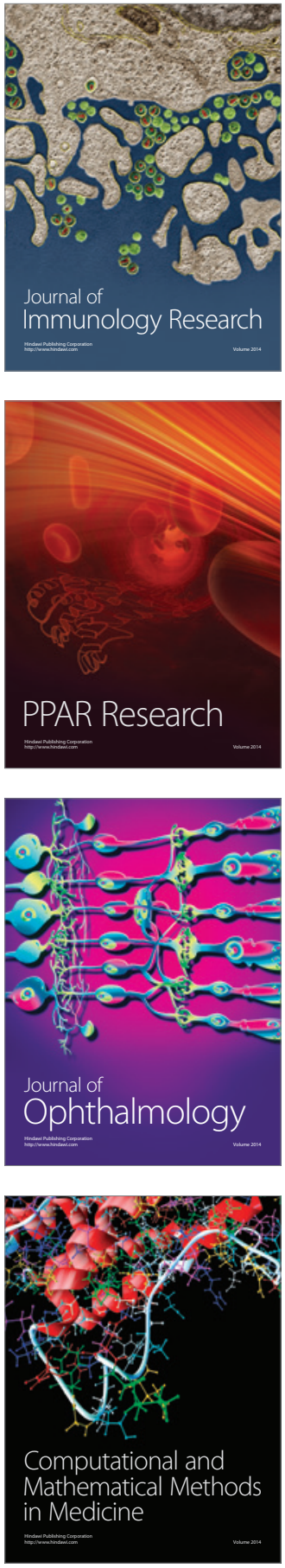

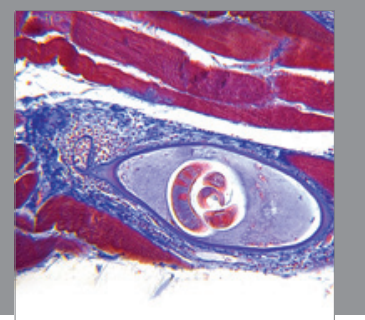

Gastroenterology

Research and Practice
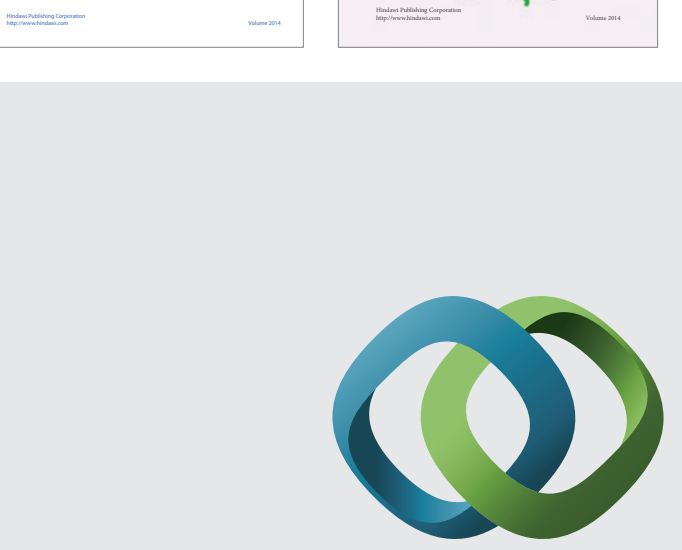

\section{Hindawi}

Submit your manuscripts at

http://www.hindawi.com
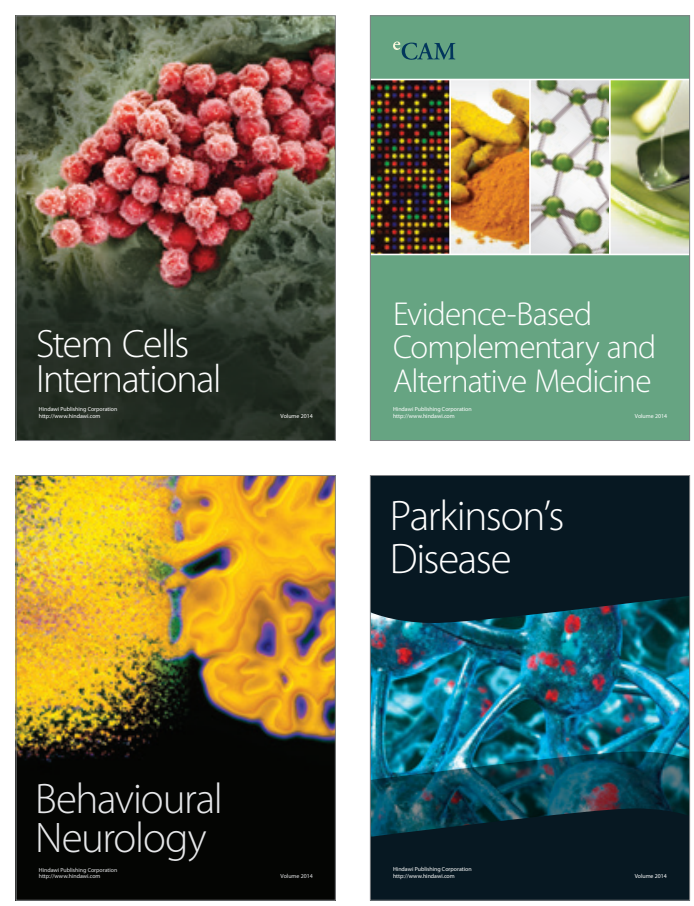

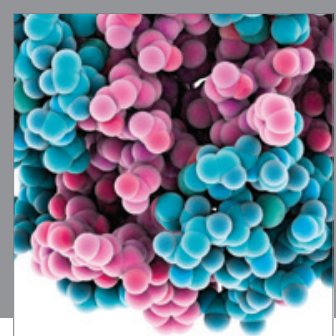

Journal of
Diabetes Research

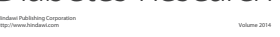

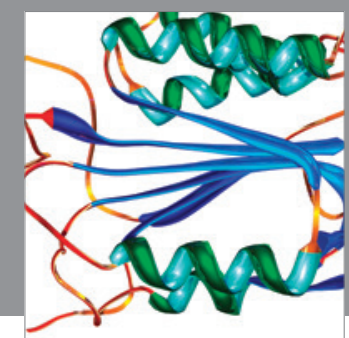

Disease Markers
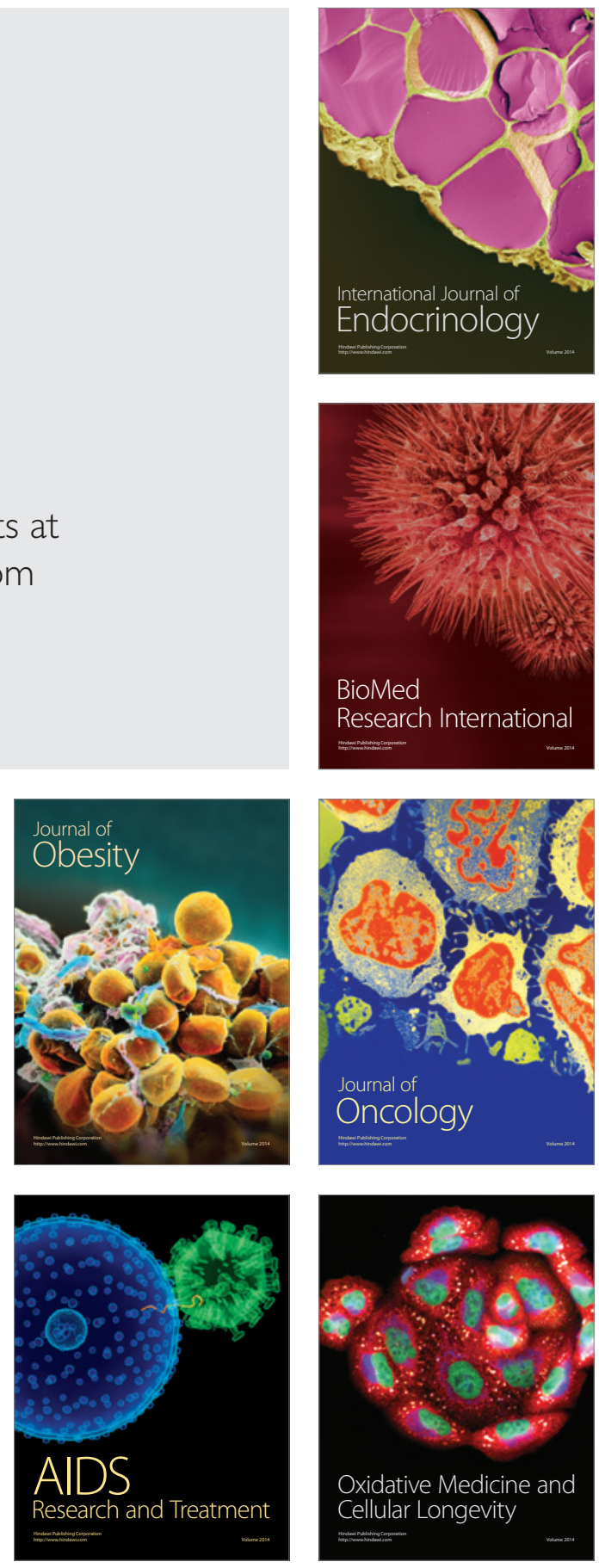